\title{
Epigenetic inactivation of PLCD1 in chronic myeloid leukemia
}

\author{
JUN-JUN SONG ${ }^{1 *}$, QIONG LIU ${ }^{2 *}$, YING LI ${ }^{1}$, ZE-SONG YANG ${ }^{1}$, LI YANG $^{3}$, TING-XIU XIANG ${ }^{4}$, \\ GUO-SHENG REN ${ }^{4}$ and JIAN-BIN CHEN ${ }^{1}$
}

\author{
Departments of ${ }^{1}$ Hematology and ${ }^{2}$ Emergency, The First Affiliated Hospital of Chongqing Medical \\ University, Chongqing; ${ }^{3}$ Chongqing Medical University, Chongqing; ${ }^{4}$ Molecular Oncology and Epigenetics \\ Laboratory, The First Affiliated Hospital of Chongqing Medical University, Chongqing, P.R. China
}

Received January 27, 2012; Accepted March 23, 2012

DOI: $10.3892 / \mathrm{ijmm} .2012 .970$

\begin{abstract}
Phospholipase C $\delta 1$ (PLCD1), is located at the important tumor suppressor locus 3p22. It encodes an enzyme that mediates regulatory signaling of energy metabolism, calcium homeostasis and intracellular movements. PLCD1 has been studied in some human solid tumors relating to the $\mathrm{CpG}$ island methylation of the gene promoter as a functional tumor suppressor. However, no such information is available in chronic myeloid leukemia (CML). In this study, we investigated PLCD1 expression in the CML K562 cell line (0/1) and 15\% (2/13) of bone marrow mononuclear cells with CML by using semi-quantitative PCR. The CpG island (CGI) methylation status of the PLCD1 promoter was detected in K562 (0/1) and 56\% (23/41) of CML patients by methylation-specific PCR (MSP), but not in the normal adult bone marrow mononuclear cells. Furthermore, the DNA demethylation agent 5'-aza-2'deoxycytidine restored the expression of PLCD1 in K562 cells. Functional studies showed that ectopic expression of PLCD1 in K562 cells was able to dramatically inhibit their colony formation and induce cell cycle G1 arrest, suggesting that PLCD1 acts as a functional tumor suppressor and may serve as a biomarker for possible early detection and prognosis of CML.
\end{abstract}

\section{Introduction}

PLCD1 (phospholipase C 81 ) belongs to a mammalian phosphoinositide specific PLC superfamily, which is a key enzymatic superfamily in the phosphoinositide metabolism system and hydrolyses phosphatidy linositol 4,5-bisphosphate [PI(4,5)P2] to generate two second messengers, inositol 1,4,5-triphosphate

Correspondence to: Dr Jian-Bin Chen, Department of Hematology, The First Affiliated Hospital of Chongqing Medical University, Chongqing, P.R. China

E-mail: cqchenjianbin2007@126.com

*Contributed equally

Key words: phospholipase C $\delta 1$, chronic myeloid leukemia, methylation, tumor suppressor gene and diacylglycerol. To date, there are six major families of PLC enzymes $(\beta, \gamma, \delta, \varepsilon, \zeta$ and $\eta)$. Among these family members, PLCD is considered to be the basic isoform and to be composed of 3 isozymes, PLCD1, D3 and D4 (1). Deletion of 3P is one of the most common genetic alterations in multiple cancers $(2,3)$. It is believed that multiple TSGs are located in this important region, such as BLU, DLEC1 and PLCD1 (4-6).

In the pathogenesis of leukemia, epigenetic silencing of TSGs is a frequent event (7-9). The methylation of several TSGs, such as estrogen receptor (ER), death-associated protein kinase (DAPK1) and protein tyrosine phosphatase receptor typer (PTPRG), have been confirmed to be associated with the development and progression of chronic myeloid leukemia (CML) (10-12). Multiple TSGs at 3p have been reported, such as BLU, DLEC1, PLCD1, FHIT, RASSFIA, CACNA2D2 (4-6,13-15) and these TSGs are involved in the pathogenesis of various tumors. Although these TSGs have been widely studied in many tumors, TSG PLCD1 with tumor-specific methylation has not been identified in CML, which would be worthy of further exploring to identify novel diagnostic biomarkers and therapeutic strategies for this tumor.

Here, we report that PLCD1 is frequently methylated in the CML K562 cell line and bone marrow samples with CML, resulting in loss of its expression at the mRNA level. Ectopic PLCD1 expression in K562 cells obviously suppressed colony formation ability and led to cell cycle arrest at G1 phase, confirming that PLCD1 is a novel functional tumor suppressor gene in CML.

\section{Materials and methods}

Cell culture and sample collection. The K562 CML cell line was obtained from the Clinical Laboratory of Chongqing Medical University (Chongqing, China) and routinely maintained in RPMI-1640 media (Gibco-BRL, USA), supplemented with $10 \%$ heat-inactivated fetal bovine serum (Gibco-BRL). We studied 41 patients [18 females, 23 males, of a median age of 46 years (range 17-80 years)] with CML and 15 healthy adults. Thirty-one patients were in chronic (CP), 3 in accelerated (AP) and 7 in blast phase (BP). The diagnosis was established according to standard morphologic and immunophenotypic criteria of WHO classification (16). The samples were collected by the Laboratory of Hematology (the 
Table I. Correlation between methylation of the PLCD1 gene and the clinical characteristics of CML patients.

\begin{tabular}{|c|c|c|c|c|}
\hline Patient parameters & $\begin{array}{l}\text { Patients with methylated } \\
\text { PLCD1 }(n=23)\end{array}$ & $\begin{array}{l}\text { Patients with unmethylated } \\
\text { PLCD1 }(n=18)\end{array}$ & Total $(n=41)$ & P-value \\
\hline
\end{tabular}

\begin{tabular}{|c|c|c|c|c|}
\hline \multicolumn{4}{|l|}{ Staging, $\mathrm{n}$} & \multirow[t]{4}{*}{0.1378} \\
\hline $\mathrm{CP}$ & 17 & 14 & 31 & \\
\hline AP & 2 & 1 & 3 & \\
\hline BP & 4 & 3 & 7 & \\
\hline Gender (male/female), $\mathrm{n}$ & $14 / 9$ & $9 / 9$ & $23 / 18$ & 0.486 \\
\hline Age (years) mean (range) & $45.74(17-80)$ & $46.90(18-75)$ & $45.99(17-80)$ & 0.843 \\
\hline WBC $\left(\times 10^{9}\right)$ mean (range) & $38.00(3.18-213)$ & $67.46(2.1-284)$ & $49.43(2.1-284)$ & 0.243 \\
\hline Hemoglobin $\left(\times 10^{9}\right)$ mean (range) & $89.09(43-155)$ & $79.00(10-133)$ & $86.01(10-155)$ & 0.724 \\
\hline Platelet counts $\left(\mathrm{x} 10^{9}\right)$ mean (range) & $273.17(11-873)$ & $422.56(21-913)$ & $352.07(11-913)$ & 0.082 \\
\hline
\end{tabular}

$\mathrm{CP}$, chronic phase; AP, accelerated phase; BP, blast phase; WBC, white blood cells.

First Affiliated Hospital of Chongqing Medical University, Chongqing, China). Informed consent was provided according to the Declaration of Helsinki.

Drug treatment. K562 cells were treated with $10 \mu \mathrm{mol} / 1$ 5'-aza2'-deoxycytidine (Aza; Sigma, USA) for 3 days and further treated with $100 \mu \mathrm{mol} / 1$ trichostatin A (TSA; Sigma) for $24 \mathrm{~h}$ as described previously $(17,18)$.

Total-RNA isolation and semi-quantitative reverse transcription (RT-PCR). Total-RNA was reversely transcribed to first-strand cDNA, using Go-Taq (Promega, USA) and random hexamer primers. GAPDH served as a control for RNA integrity. PLCD1 expression was analyzed by PCR, primers used were PLCD1-F, 5'-TGTCGCTACTCAAGTGAGTC-3' and PLCD1-R, 5'-AGT CCTCCTGAACTTGTAG-3'. GAPDH-F, 5'-ATCTCTGCCC CCTCTGCTGA-3' and GAPDH-R, 5'-GATGACCTTGCCCA CAGCCT-3'. The PCR thermocycling conditions included initial denaturation at $95^{\circ} \mathrm{C}$ for $10 \mathrm{~min}$, followed by 35 cycles (for PLCD1) or 23 cycles (for GAPDH) of reaction $\left(95^{\circ} \mathrm{C}\right.$ for $30 \mathrm{sec}, 55^{\circ} \mathrm{C}$ for $30 \mathrm{sec}$ and $72^{\circ} \mathrm{C}$ for $30 \mathrm{sec}$ ), using Go-Taq polymerase (Promega), with a final extension at $72^{\circ} \mathrm{C}$ for $10 \mathrm{~min}$.

DNA bisulfite treatment and methylation analysis. DNA was extracted from CML and healthy adult bone marrow samples by standard method (Zymo Research, USA). Bisulfite modification of DNA and methylation status in the $\mathrm{CpG}$ island of PLCD1 promoter were carried out as described previously $(19,20)$. PLCD1 primers detecting methylation or unmethylation alleles of the promoter were PLCD1-m1, 5'-AATGATA GGGTTCGCGGTTC-3' and PLCD1-m2, 5'-CCCGAACCAA CGAACGCG-3' for methylated; PLCD1-u1, 5'-GTAATGA TAGGGTTTGTGGTTT-3' and PLCD1-u2, 5'-TAACCCAAA CCAACAAACACA-3' for unmethylated. Methylation-specific PCR (MSP) was done for 40 cycles using Ampli Taq-Gold (methylation specific primer: annealing temperature $60^{\circ} \mathrm{C}$, unmethylation specific primer: annealing temperature $58^{\circ} \mathrm{C}$ ). MSP primers were tested previously for not amplifying any unbisulfite DNA and the specificity of MSP was further confirmed by any direct sequencing of some PCR products. PCR reactions were resolved on a $2 \%$ agarose gel.

Cell transfection and colony formation assays. K562 cells were plated in a 6-well plate and transfected with PLCD1 expression plasmid pcDNA3.1 or the empty vector pcDNA3.1 $(0.8 \mu \mathrm{g}$ each) respectively, using FuGENE ${ }^{\circledR} 6$ (Roche Diagnostics, Mannheim, Germany). Forty-eight hours post-transfection, cells were re-plated into a 6-well plate with G418 selection $(0.5 \mu \mathrm{g} / \mu \mathrm{l})$. The selective medium was replaced every 3 days. After 2 weeks, stable PLCD1 expression clones were selected for further study. Total-RNA was extracted and analyzed by RT-PCR to confirm the ectopic expression of PLCD1.

Stable PLCD1 transfectants and the vector cells were suspended in RPMI-1640 containing 1\% methylcellulose, $10 \%$ fetal bovine serum (FBS) and $0.5 \mathrm{mg} / \mathrm{ml} \mathrm{G418} \mathrm{in} \mathrm{a} 6$-well plate. After 2 weeks, surviving colonies $(\geq 50)$ were counted under a light microscope. All the experiments were performed 3 times in triplicate wells.

Cell cycle analysis. The stable PLCD1 expression cells (PLCD1K562) or Vector-K562 cells were cultured in RPMI-1640 medium and $10 \%$ FBS with G418 (0.2 mg/ml). These cells were harvested and fixed in ice-cold $75 \%$ ethanol for $1 \mathrm{~h}$. The cell cycle profiles were assayed by the Elite ESP flow cytometer and data were analyzed with the CellQuest software (BD Biosciences, USA).

Protein extraction and western blot analysis. After washing the cells with PBS for 3 times, PLCD1-K562 or Vector-K562 cells were harvested and lysed in RTPA buffer containing $0.1 \mathrm{mg} / \mathrm{ml}$ of PMSF as final concentrations. Western blot analysis was performed according to the standard protocol as described (13). Anti-PLCD1 rabbit polyclonal antibody (1:700; GeneTex, Inc., USA) and anti-GAPDH mouse monoclonal antibody (1:1,000; Epitomics, Inc., USA) were used.

Statistical analysis. All statistical calculations were done using SAS version 9.1 for windows (SAS Institute, Inc., Cary, NC, USA). The correlation between the frequency of PLCD1 


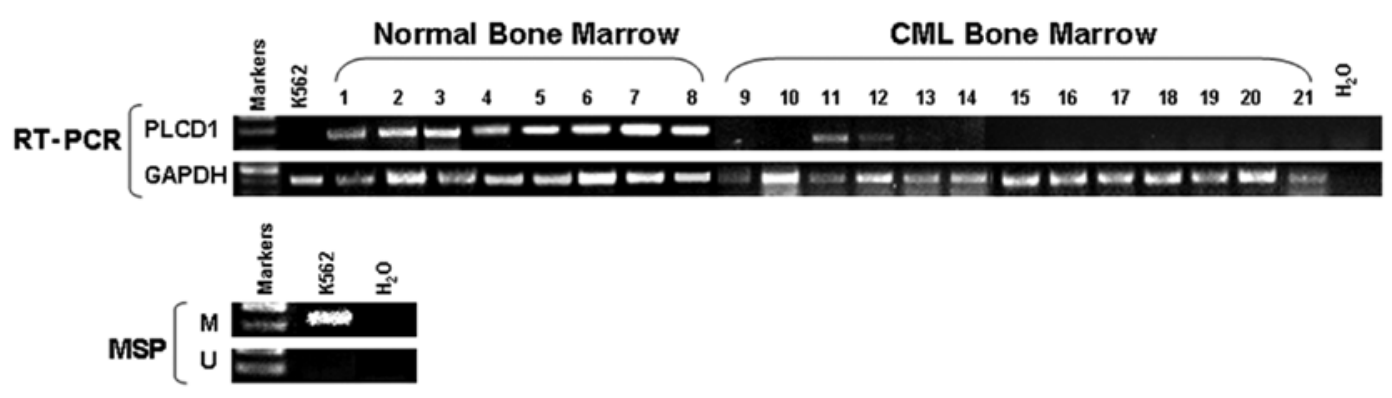

Figure 1. Downregulation of PLCD1 by promoter methylation in CML K562 cell line. PLCD1 expression and methylation status in K562 cells was determined by semi-quantitative RT-PCR and MSP. PLCD1 expression in human normal and CML samples was determined by semi-quantitative RT-PCR with GAPDH as a control. M, methylated; $\mathrm{U}$, unmethylated.

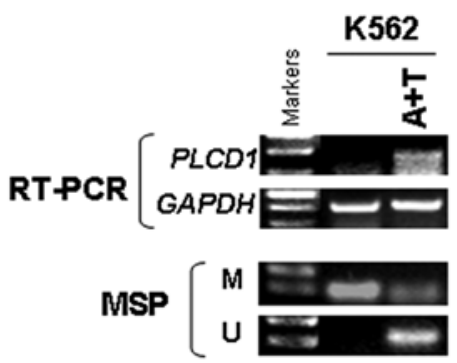

Figure 2. Pharmacological demethylation using Aza together with TSA reactivating the silenced PLCD1 in K562 cells. A+T, Aza and TSA treatment.

promoter methylation and age, white blood cells, hemoglobin level and platelet counts was analyzed with Student's t-test. Student's t-test was also used for the difference in subgroups (cell cycle analysis and colony formation assay). Chi-square test was carried out to compare the correlation between frequency of PLCD1 promoter methylation and clinical stages, correlation between the frequency and gender was analyzed with Fisher's exact test. P-value $<0.05$ was considered to be significant.

\section{Results}

PLCD1 downregulation and promoter hypermethylation in K562 cells. To examine if PLCD1 is downregulated in K562 cells, we first examined the expression in K562 cells, 8 normal adult bone marrow samples and $13 \mathrm{CML}$ samples with semiquantitative RT-PCR. PLCD1 was silenced in K562 cells and 11/13 (85\%) CML bone marrow samples while readily detected in normal adult bone marrow samples (0/8) (Fig. 1).

We further analyzed the methylation status of PLCD1 to determine whether downregulation in K562 cells is caused by hypermethylation in its promoter region. MSP using methylation- or unmethylation-specific primers was done to investigate the methylation status of PLCD1. PLCD1 CGI was methylated in K562 cells, while no methylation was detected in normal adult bone marrow (Fig. 1), showing that the silence of PLCD1 expression in K562 cells was correlated with its methylation status.

Pharmacological demethylation reactivates the silenced $P L C D 1$. To evaluate the effect of promoter CGI methylation on the expression of PLCD1, K562 cells were treated with a DNA methytransferase inhibitor, 5'-azacytidine for 3 days together with a histone deacetylase inhibitor, TSA for 1 day. As shown in Fig. 2, PLCD1 in mRNA expression was dramatically induced after the treatment, this reactivation was associated with an increase of unmethylated alleles and decrease of methylated alleles of the PLCD1 promoter, as assessed by MSP.These results show a direct link between CGI methylation and PLCD1 silencing.

Frequent PLCD1 methylation in various phases of CML. In view of the effect of promoter region methylation on the expression of PLCD1 in K562 cells, we proceeded to determine the methylation status of CML and normal adult bone marrow samples. As summarized in Fig. 3, aberrant methylation was detected in 23/41 (56\%) CML samples (17/41 in the stage of CP, 2/41 in the stage of AP, 4/41 in the stage of $\mathrm{BP})$, but not in normal adult samples $(0 / 15)$. The frequencies of PLCD1 promoter methylation in CML patients in CP, AP and BP are shown in Table I. Thus, promoter methylation of PLCD1 is a frequent and tumor-specific event in CML. The correlation between PLCD1 promoter methylation and clinical stage, age, gender, white blood cells (WBC), hemoglobin (Hb) and platelet counts (PLT) are shown in Table I.

Ectopic expression of PLCD1 inhibites tumor cell clonogenicity and induces $G 1$ cell cycle arrest. To evaluate the role of PLCD1 as a TSG in CML, we thus sought to establish whether ectopic expression of PLCD1 could inhibit tumor cell clonogenicity. The expression vector encoding fulllength PLCD1 or vector alone were transfected into K562 cells, in which PLCD1 was fully silenced by methylation. After G418 seletion for 2 weeks, stable overexpression of PLCD1 as shown by RT-PCR and western blot analysis, was successfully obtained (Fig. 4C). Colony formation assay (Fig. 4A) was used to evaluate the suppressor function of PLCD1 in vector or PLCD1-transfected cells. Ectopic expression of PLCD1 dramatically reduced the colony formation efficiencies of K562 cells (Fig. 4B) down to $40-50 \%$ of vector controls, $\mathrm{P}<0.05$ ), indicating that PLCD1 was a functional TSG in K562 cells.

To explore the mechanism by which PLCD1 suppresses colony formation, we investigated the effect of PLCD1 on cell cycle distribution by flow cytometry. The percentage of cells in G1 phase was increased in PLCD1-transfected cells compared 

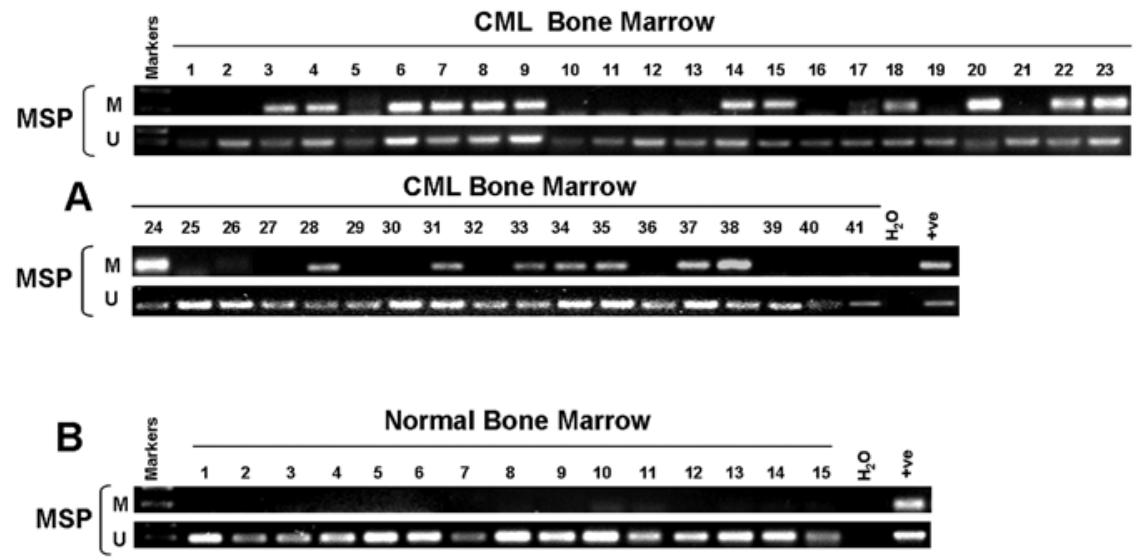

Figure 3. PLCD1 CpG methylation in CML and normal adult bone marrow samples. (A) CML bone marrow samples. (B) Normal adult bone marrow samples. $\mathrm{M}$, methylation; U, unmethylation.

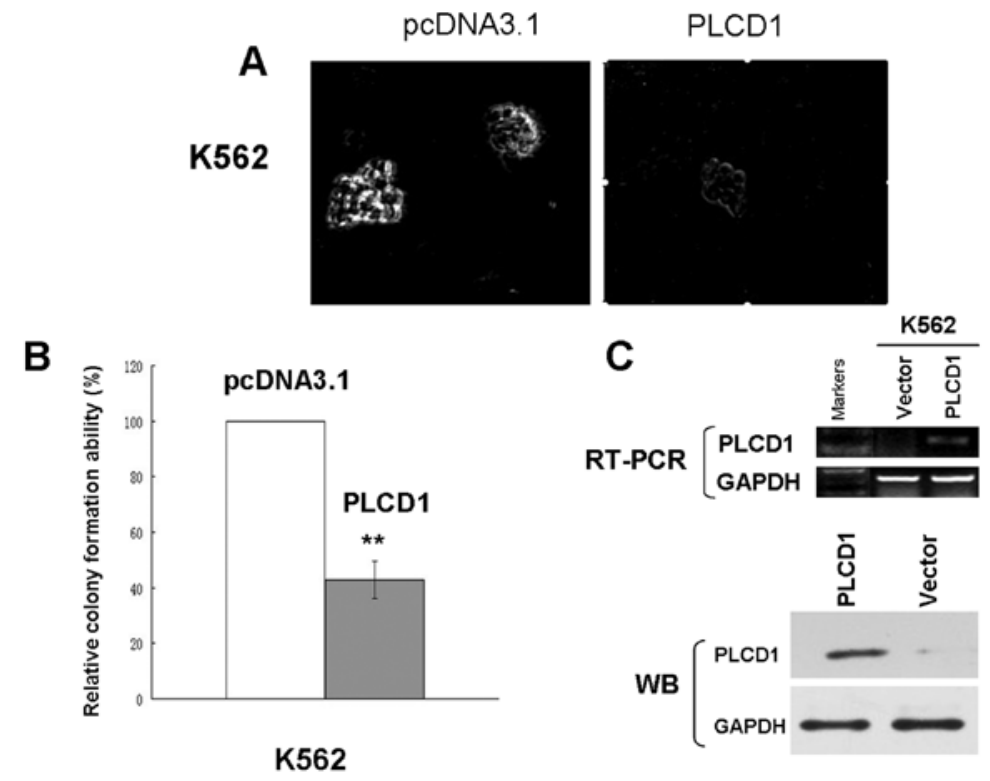

Figure 4. Ectopic expression of PLCD1 inhibited tumor cell clonogenicity. (A) Representative inhibition of colony formation in soft agar culture by PLCD1. (B) Quantitative analysis of colony formation. The numbers of G418 selection colonies in vector-transfected cell line were set to $100 \%$, values are mean \pm SD, $\mathrm{P}$-values were calculated using Student's-test. ${ }^{* *}$ Significant statistical difference $(\mathrm{P}<0.05)$. Independent experiments were performed in triplicate wells 3 times. (C) Ectopic expression of PLCD1 in the transcript level in transfected K562 cells was confirmed by RT-PCR and WB. WB, western blot analysis.

to vector $\mathrm{K} 562$ cells $(\mathrm{P}<0.05)$, indicating that the effect of PLCD1 was likely to be dependent on the cell cycle (Fig. 5). Thus, taken these results together, PLCD1 indeed has growth inhibitory activity and was a functional TSG in CML.

\section{Discussion}

Multiple TSGs at $3 p$ have been reported, such as BLU, DLEC1, PLCD1, FHIT, RASSFIA, CACNA2D2 (4-6,13-15), and these TSGs are involved in the pathogenesis of various tumors such as gastrointestinal tract, lung, nasopharynx, esophagus, kidney, breast and cervix. Although these TSGs have been widely studied in many tumors, TSG PLCD1 with tumor-specific methylation has not been identified in CML.

In the present study, we found that PLCD1 was downregulated in K562 cells and in 85\% CML bone marrow samples, but widely expressed in normal adult bone marrow samples.
Our study showed that promoter CGI methylation of PLCD1 in K562 cells and 56\% CML samples, and pharmacological demethylation could restore PLCD1 expression, suggesting that promoter CGI methylation plays an important role in the inactivation of PLCD1 during the tumorigenesis of CML.

Our present study showed that ectopic expression of PLCD1 in K562 cells significantly suppressed their colony formation which was able to arrest tumor cells at cell cycle in K562. Fu et al (21) demonstrated that PLCD1 exerted its tumor-suppressive function through inhibiting cell cycle progression from G1 to S-phase in ESCC, which is consistent with our findings. Thus, PLCD1 is a functional TSG in CML.

PLCD1 methylation was found to be significantly correlated with lymph node metastasis in breast cancer (18), ESCC (21) and high-stage gastric tumor (6), indicating that PLCD1 has been identified as a TSG and can be a potentical biomarker in these tumors. The high incidence of this tumor-specific 

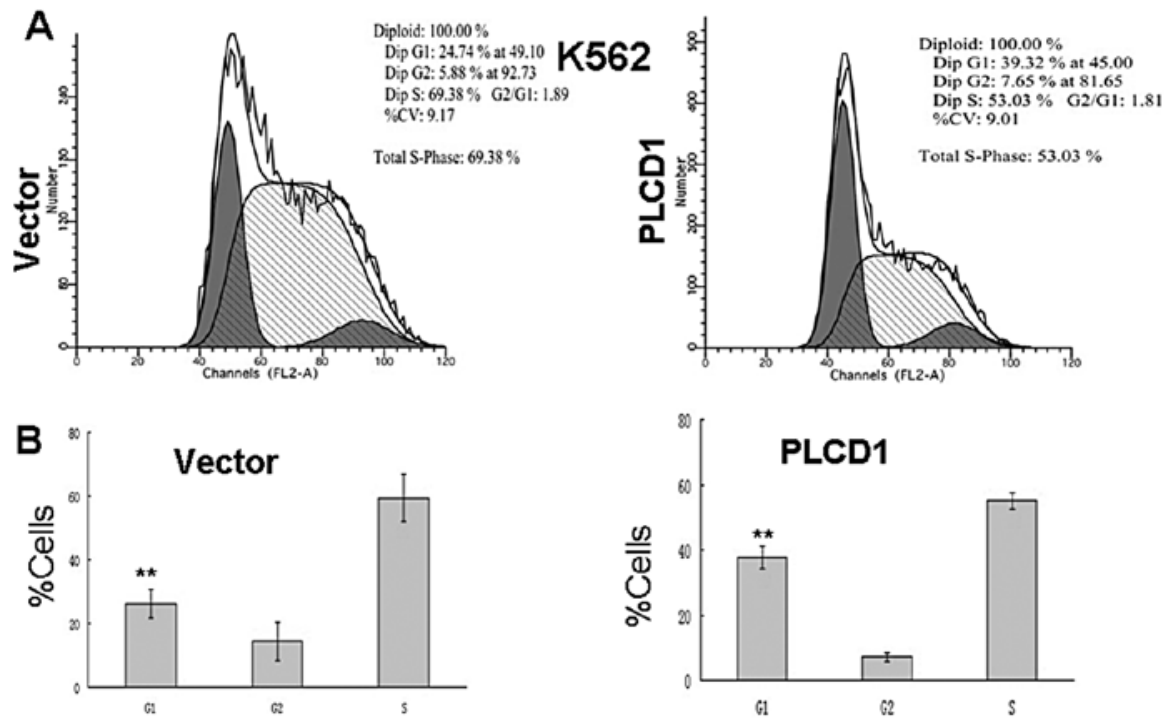

Figure 5. PLCD1 induces cell cycle arrest at the G1 phase in K562 cells. (A) Representative distribution of cell cycles in K562 cells transfected with or without PLCD1 by fluorescence-activated cell sorting analysis. (B) Analysis of K562 cells transfected with or without PLCD1 distributing in different phases of cell. Results are presented as the mean $\pm \mathrm{SD}(\mathrm{n}=4)$. Significant statistical difference, $\mathrm{P}<0.05$ (Student's t-test).

molecular marker in CML suggest that it might be a clinically useful marker of disease. PLCD1 is a relevant new player in the pathogenesis of CML and suggests that it as a potiential target for diagnostic and therapeutic applications. There were no significant correlations between the methylation status of PLCD1 promoter and the clinical features, such as clinical stages, age, gender, hemoglobin level, white blood cells $(\mathrm{WBC})$ and platelet counts $(\mathrm{P}>0.05)$. Our results suggest that the methylation of PLCD1 promoter might occur in the early stage of CML development. However, the clinical impact of PLCD1 inactivation in CML is still unknown, further studies of possible clinicopathological relevance of PLCD1 methylation and its potiential diagnostic and prognostic implications in patients with CML are needed, such as chromosomal abnormalities and BCR/ABL transcript. Other mechanisms besides DNA methylation such as histone modification might also be involved in the regulation of PLCD1 expression. More cases should be studied to determine the impact of PLCD1 methylation on the regulation of transcription.

In summary, we found that PLCD1 is frequently silenced by promoter methylation in CML in a tumor-specific manner, which could be activated by pharmacological demethylation. Ectoptic expression of PLCD1 suppresses CML cells clonogenicity and leads to the disruption of cycle progression at G1 phase in CML, supporting that PLCD1 is a TSG in this tumor. It would be worthy of further exploring the possible use of PLCD1 methylation as an epigenetic biomarker for future molecular diagnosis.

\section{Acknowledgements}

We thank Professor Qian Tao (the Chinese University of Hong Kong, Hong Kong, China), for generously providing technical assistance and the great help with the whole design of the experiment. We also thank the Molecular Oncology and Epigenetics Laboratory (the First Affiliated Hospital of Chongqing Medical University) for technical assistance and Laboratory of Hematology (the First Affiliated Hospital of Chongqing Medical University) for the bone marrow samples.

\section{References}

1. Rhee SG: Regulation of phosphoinositide-specific phospholipase C. Annu Rev Biochem 70: 281-312, 2001.

2. Hesson LB, Cooper WN and Latif F: Evaluation of the 3p21.3 tumor-suppressor gene cluster. Oncogene 26: 7283-7301, 2007.

3. Zabarovsky ER, Lerman MI and Minna JD: Tumor suppressor genes on chromosome $3 p$ involved in the pathogenesis of lung and other cancers. Oncogene 21: 6915-6935, 2002.

4. Qiu GH, Tan LK, Loh KS, et al: The candidate tumor suppressor gene BLU, located at the commonly deleted region $3 \mathrm{p} 21.3$, is and E2F-regulated, stress-responsive gene and inactivated by both epigenetic and genetic mechanisms in nasopharyngeal carcinoma. Oncogene 23: 4793-4806, 2004

5. Qiu GH, Salto-Tellez M, Ross JA, et al: The tumor suppressor gene DLEC1 is frequently silenced by DNA methylation in hepatocellular carcinoma and induces G1 arrest in cell cycle. J Hepatol 48: 433-441, 2008.

6. Hu XT, Zhang FB, Fan YC, et al: Phospholipase Cdeltal is a novel 3p22.3 tumor suppressor involved in cytoskeleton organization, with its epigenetic silencing correlated with high-stage gastric cancer. Oncogene 28: 2466-2475, 2009.

7. Jones PA and Baylin SB: The epigenomics of cancer. Cell 128: 683-692, 2007.

8. Baylin SB and Herman JG: DNA hypermethylation in tumorigenesis: epigenetics joins genetics. Trend Genet 16: 168-174, 2000.

9. Esteller M: Aberrant DNA methylation as a cancer-inducing mechanism. Annu Rev Pharmacol Toxicol 45: 629-656, 2005.

10. Issa JP, Zehnbauer BA, Civin $\mathrm{Cl}$, et al: The estrogen receptor $\mathrm{CpG}$ island is methylated in most hematopoietic neoplasms. Cancer Res 56: 973-977, 1996.

11. Qian J, Wang YL, Lin J, Yao DM, Xu WR and Wu CY: Aberrant methylation of the death-associated protein kinase 1(DAPK1) $\mathrm{CpG}$ inland in chronic myeloid leukemia. Eur J Haematol 82: 119-123, 2009.

12. Della Peruta M, Martinelli G, Moratti E, et al: Protein tyrosine phosphatase receptor type $\gamma$ is a functional tumor suppressor gene specifically downregulated in chronic myeloid leukemia. Cancer Res 70: 8896-8906, 2010.

13. Ohta M, Inoue H, Cotticelli MG, et al: The FHIT gene, spanning the chromosome 3p14.2 fragile site and renal carcinoma-associated $\mathrm{t}(3 ; 8)$ breakpoint, is abnormal in digestive tract cancers. Cell 84: 587-597, 1996. 
14. Dammann R, Li C, Yoon JH, Chin PL, Bates S and Pfeifer GP Epigenetic inactivation of a RAS association domain family protein from the lung tumor suppressor locus 3p21.3. Nat Genet 25: 315-319, 2000

15. Ji L, Nishizaki M, Gao B, et al: Expression of several genes in the human chromosome $3 \mathrm{p} 21.3$ homozygous deletion region by an adenovirus vector results in tumor suppressor activities in vitro and in vivo. Cancer Res 62: 2715-2720,2002.

16. Vardiman JW, Harris NL and Brunning RD: The World Health Organization (WHO) classification of the myeloid neoplasms. Blood 100: 2292-2302, 2002

17. Wang Y, Li J, Cui Y, et al: CMTM3, located at the critical tumor suppressor locus $16 \mathrm{p} 22.1$, is silenced by $\mathrm{CpG}$ methylation in carcinomas and inhibits tumor cell growth through inducing apoptosis. Cancer Res 69: 5194-5201, 2009.
18. Xiang T, Li L, Fan Y, et al: PLCD1 is a functional tumor suppressor inducing $\mathrm{G} 2 / \mathrm{M}$ arrest and frequently methylated in breast cancer. Cancer Biol Ther 10: 520-527, 2010.

19. Tao Q, Huang H, Geiman TM, Lim CY, Fu L, Qiu GH and Robertson KD: Defective de novo methylation of viral and cellular DNA sequences in ICF syndrome cells. Hum Mol Genet 11: 2091-2102, 2002.

20. Tao Q, Swinnen LJ, Yang J, Srivastava G,Robertson KD and Ambinder RF: Methylation status of the EpsteinBarr virus major latent promoter $\mathrm{C}$ in iatrogenic $\mathrm{B}$ cell lymphoproliferative disease. Application of PCR-based analysis. Am J Pathol 155: 619-625, 1999.

21. Fu L, Qin YR, Xie D, et al: Characterization of a novel tumorsuppressor gene PLC delta1 at 3p22 in esophageal squamous cell carcinoma. Cancer Res 67: 10720-10726, 2007. 